\title{
GoSPo: a goniospectropolarimeter to assess reflectance, transmittance, and surface polarization as related to leaf optical properties
}

\author{
Reisha D. Peters $\odot{ }^{\mathrm{a}, *}$ Simone R. Hagey $\oplus^{\mathrm{b}}{ }^{\mathrm{b}}$ and Scott D. Noble $\odot^{\mathrm{c}}$ \\ ${ }^{a}$ University of Saskatchewan, Department of Chemical and Biological Engineering, \\ Saskatoon, Canada \\ ${ }^{b}$ University of Saskatchewan, Department of Physics and Engineering Physics, \\ Saskatoon, Canada \\ ${ }^{c}$ University of Saskatchewan, Department of Mechanical Engineering, Saskatoon, Canada
}

\begin{abstract}
Visible-near infrared (VIS-NIR) spectral data are widely used for remotely estimating a number of crop health metrics. In general, these indices and models do not explicitly account for leaf surface characteristics, which themselves can be indicators of plant status or environmental responses. To explicitly include leaf surface characteristics, data are required linking optical properties to surface characteristics. We present the design and experimental validation of a goniospectropolarimeter (GoSPo) that combines the capabilities of a spectrometer, goniometer, and polarimeter. GoSPo was designed with the objective of studying the relationships between leaf surface characteristics and the resulting light reflectance, transmission, and polarization as functions of both direction and VIS-NIR spectra. Using six motors, a pneumatic system, two spectrometers, and a combination of lenses, polarizers, and mirrors, GoSPo can examine a leaf from a particular angle, approximate hemispherical transmittance and reflectance (with root-mean-square error values of 0.0189 and 0.0216 for reflectance and transmittance, respectively, compared to a spectrophotometer and integrating sphere), and obtain spectral polarization measurements without disrupting the sample between measurements. The data collected with GoSPo will aid in model development for remote sensing applications. (C) The Authors. Published by SPIE under a Creative Commons Attribution 4.0 Unported License. Distribution or reproduction of this work in whole or in part requires full attribution of the original publication, including its DOI. [DOI: 10.1117/1.JRS.14.047505]
\end{abstract}

Keywords: reflectance; transmittance; goniometer; polarization; remote sensing; leaves.

Paper 200452 received Jun. 15, 2020; accepted for publication Nov. 23, 2020; published online Dec. 10, 2020.

\section{Introduction}

The spectral reflectance of plant leaves has been measured and modeled for decades to inform remote methods for monitoring vegetation health and plant status. Many models and vegetation indices have been developed relating spectral reflectance characteristics to leaf pigment content and changes related to leaf internal structure. However, the wide variability in surface property contributions to leaf reflectance is not explicitly or significantly accounted for in the most prevalent models. ${ }^{1}$ Models based on directional measurements use hemispherical leaf measurements in calibration and development. This method is useful for analysis of individual leaf measurements in a laboratory setting and can be applied when averaging canopy angle, but as the spatial resolution of data improves and close-range multiangular modeling of leaf surface-light interactions have more potential value. Some recent work has examined introducing directional effects, modeled as facets. ${ }^{2-5}$ Other works have looked at qualitative correlation of surface properties (wax loading, trichomes, and cell cap structure) to reflectance characteristics ${ }^{6,7}$ and identified an increase in specular light reflectance with optically smooth and waxy leaves.

Reflectance is expressed as the fraction of the light reflected from an object relative to the light incident upon it. ${ }^{8}$ Reflectance can be further categorized as being specular or diffuse.

*Address all correspondence to Reisha D. Peters, reisha.peters@usask.ca 
For leaves, most diffuse reflectance arises from internal scattering. ${ }^{6,9,10}$ Transitions in index of refraction within the leaf, such as between cell walls and intercellular air pockets, will refract or reflect the light rays in a new direction. After multiple scattering, light exits the leaf in randomized directions and is measured as diffuse reflectance or transmittance. ${ }^{6,9,10}$ In contrast, light that is reflected from the surface of the leaf, at the boundary between air and cuticle, is a combination of specular and diffuse reflectance. ${ }^{7,10}$ Specular reflectance is what makes a leaf appear shiny or glossy and is affected by the surface properties of the leaf., ${ }^{4,7}$ The magnitude and angular distribution of the specular reflectance can also be used in estimating the index of refraction. ${ }^{4}$ There is evidence that by separating surface reflectance from the subsurface reflectance of leaves, more can be determined about the physical characteristics of the plant.

Light reflected at the surface never enters the leaf, so it does not interact with internal leaf tissue, but may contain information about the surface properties. In contrast, diffusely reflected light from subsurface interactions has been influenced by leaf water, pigment, and structure. ${ }^{10,11}$ If the surface component of leaf reflectance is well characterized, information about the interior of the leaf may be extracted with greater accuracy.

Separating the surface and subsurface reflectance is difficult as the measured surface reflectance is a combination of specular reflection and diffuse reflection arising from the optically rough, waxy outer layer of the cuticle. ${ }^{9,10}$ Diffusely reflected light from surface and internal scattering cannot be differentiated, ${ }^{9}$ but directionality and polarization can aid in separating the specular reflectance from the surface and the subsurface component from diffuse scattering. The degree of polarization can indicate the roughness of a leaf's surface and leads to identification of the surface phenotype. ${ }^{7}$

The standard approach for acquiring hemispherical reflectance and transmittance measurements uses an integrating sphere. However, it has been hypothesized that the most important variable for understanding polarized reflectance from plant matter is the angle between the illumination and observation directions. ${ }^{10}$ This specifies the need for an instrument capable of measuring bidirectional reflectance and transmittance with associated polarization measurements. Resolving angular distributions and surface polarization measurements cannot be obtained using an integrating sphere.

Previous studies have used goniometers equipped with polarizing filters to separate specular and diffuse light. Vanderbilt et al. ${ }^{12}$ used a polarizing photometer with the illumination angle and viewing angle fixed at $55 \mathrm{deg}$ to investigate light scattering properties of leaves. Combes et al. ${ }^{2}$ were able to design an apparatus to estimate bidirectional reflectance distribution function (BRDF) and bidirectional transmittance distribution function (BTDF), respectively and integrate hemispherical reflectance and transmittance, but limited their experiment to 400 to $800 \mathrm{~nm}$ and did not consider polarized and nonpolarized reflectance separately. Bousquet et al. ${ }^{4,5}$ developed a five-parameter model to simulate spectral and bidirectional reflectance using the apparatus of Combes et al., but were again limited in wavelength range. Comar et al. ${ }^{13}$ focused on the differences in BRDF of monocots when illumination is parallel or perpendicular to the direction of the leaf veins. This study was able to confirm more specular reflection with perpendicular illumination and discussed the importance of the directionality of surface roughness. ${ }^{13}$

The purpose of the goniospectropolarimeter (GoSPo) is to provide polarized spectral measurements at various illumination and reflectance angles, with the overarching goal of streamlining crop phenotyping and surveillance. GoSPo was designed to aid in data collection for leaf characteristic modeling at the single-leaf scale. GoSPo achieves some of the same goals of the previously designed apparatus, but expands the spectral range further into the near infrared (NIR) and allows for both polarized and nonpolarized measurements of both reflectance and transmittance without needing to reposition the sample.

\section{Description of the Apparatus}

The GoSPo integrates the capabilities of a goniometer, spectrometer, and polarimeter. In contrast to common field goniometer designs in which the sample is stationary, ${ }^{14,15}$ the light source on GoSPo is stationary and both the sample and sensor head move to achieve various measurement geometries. A schematic illustrating major optical components and measurement angles in the 


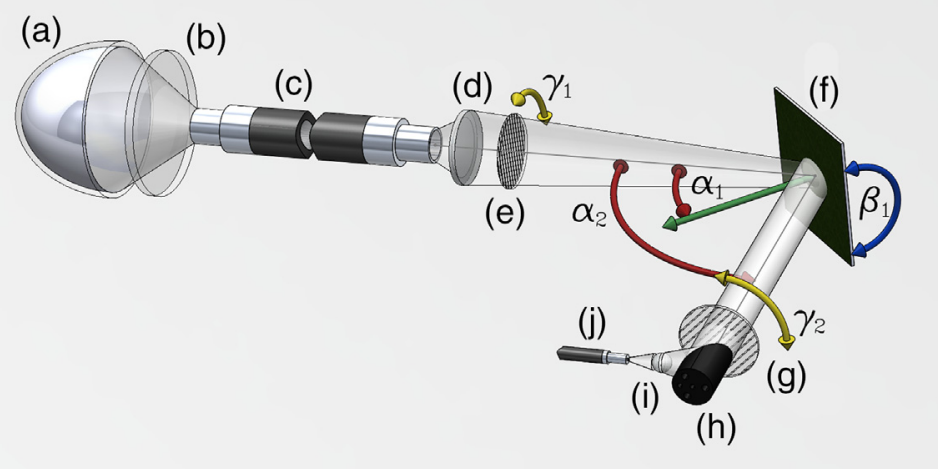

Fig. 1 Schematic of GoSPo geometry and degrees of freedom in the instrument frame of reference. (A) A tungsten-halogen lamp with aluminized elipsodic reflector; (B) heat-absorbing window; (C) quartz fiber-optic lightguide; (D) focusing lens; (E) polarizer with rotation $\gamma_{1} \in\{0 . .90$ deg $\}$; (F) target (sample or reference); $(\mathrm{G})$ analyzer with rotation $\gamma_{2} \in\{0 . .90 \mathrm{deg}\} ;(\mathrm{H})$ off-axis parabolic mirror; (I) ball lens; (J) fiber-optic cable bifurcating and terminating at the spectrometers (not shown). The principal plane is horizontal, defined by the reference axis formed by $D$ and $F$, and the axis formed by $F$ and the sensor head $(G)-(J)$ that revolves around the target $\left(\alpha_{2} \in\{6.55 \ldots 353.45 \mathrm{deg}\}\right.$. The angle of incidence is defined by rotating the sample $\left(\alpha_{1} \in\right.$ $\{-90 \ldots 90 \mathrm{deg}\})$ around the central vertical axis of the instrument. The target can be rotated around an axis in the principal plane $\left(\beta_{1}\right)$. However, this is not considered in this paper.

instrument frame of reference is shown in Fig. 1. The principal plane is horizontal, formed by the path of the sensor head (items G-J in Fig. 1) around the sample (item F) and the incident illumination ray. Angles $\alpha_{1}$ and $\alpha_{2}$ are the angles of the sample normal vector and sensor head position, respectively, in the principal plane and measured from the incident ray. In the case where the sample normal vector is parallel to the principal plane (i.e., $\beta_{1}=0 \mathrm{deg}$ )

$$
\begin{gathered}
\theta_{I}=-\alpha_{1}, \\
\theta_{E}=\alpha_{2}-\alpha_{1},
\end{gathered}
$$

and

$$
\phi_{I}=\phi_{E}=0 \mathrm{deg},
$$

where $\theta$ and $\phi$ represent the zenith and azimuth angles, respectively, in a sample frame of reference, and subscripts $I$ and $E$ indicate incident and exitant light, respectively. With azimuth angles equal to $0 \mathrm{deg}$, all zenith angles are in the principal plane with reference to the sample normal. Angles of $\theta_{I}$ range between $\pm 90 \mathrm{deg}$. Angles of $\theta_{E}$ in the ranges of $0 \mathrm{deg}$ to $90 \mathrm{deg}$ and $270 \mathrm{deg}$ to $360 \mathrm{deg}$ correspond to light reflected from the sample, while transmitted light corresponds to $\theta_{E}$ angles between $90 \mathrm{deg}$ and $270 \mathrm{deg}$. By rotating the sample normal out of the instrument principal plane (i.e., $\beta_{1} \neq 0 \mathrm{deg}$ ), nonzero azimuth angle geometries can be acheived; these are not considered in this paper.

Two spectrometers are used to obtain an operating range from 400 to $1600 \mathrm{~nm}$. A BLUEWave miniature spectrometer ( 350 to $1150 \mathrm{~nm}$, StellarNet, Tampa, Florida) is used in the visible (VIS) range, and a DWARF-Star miniature spectrometer $(900$ to $1700 \mathrm{~nm}$, StellarNet, Tampa, Florida) is used for the near-infrared region.

A tungsten-halogen lamp with an aluminized ellipsoidal reflector (International Light Technologies Inc., Peabody, Massachusetts) is used as the light source via a 0.5 -in. diameter fiber optic lightguide, allowing for the light source to be easily exchanged for different applications. A $100-\mathrm{mm}$ focal length, 25-mm diameter plano-convex lens is used to focus the output of the light source to an 8-mm diameter spot on the center of the leaf sample. Optionally mounted on the optical fiber port is a polarizing filter that can be rotated from 0 deg to $90 \operatorname{deg}\left(\gamma_{1}\right)$ via a servo motor. 
Light reflected/transmitted from the sample is collected by a $90 \mathrm{deg}$, off-axis parabolic mirror in the sensor head assembly and directed toward a ball lens coupled to a $600-\mu \mathrm{m}$, bifurcated optical fiber from StellarNet (Fig. 2). This fiber allows both spectrometers to obtain simultaneous measurements. As with the light-source aperture, the sensor head (Fig. 2) has a servo motorcontrolled optional polarizing filter (analyzer "G" in Fig. 1) that can be adjusted between 0 deg and $90 \operatorname{deg}\left(\gamma_{2}\right)$.

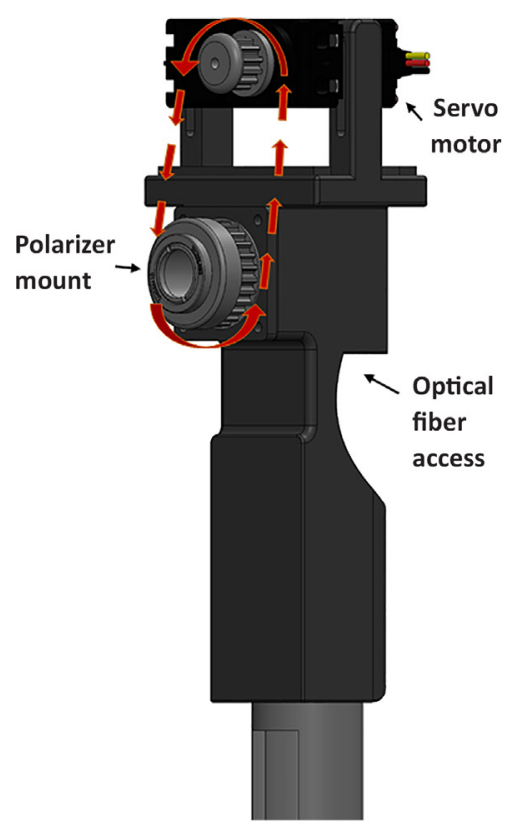

(a)

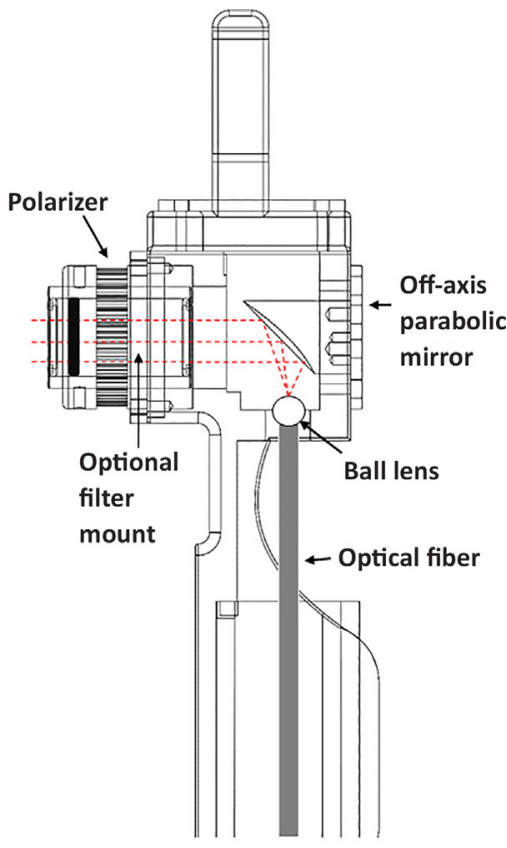

(b)

Fig. 2 (a) CAD model of the sensor head of the GoSPo and (b) a cross section illustrating the optical components inside.



Fig. 3 Full model of the GoSPo. 


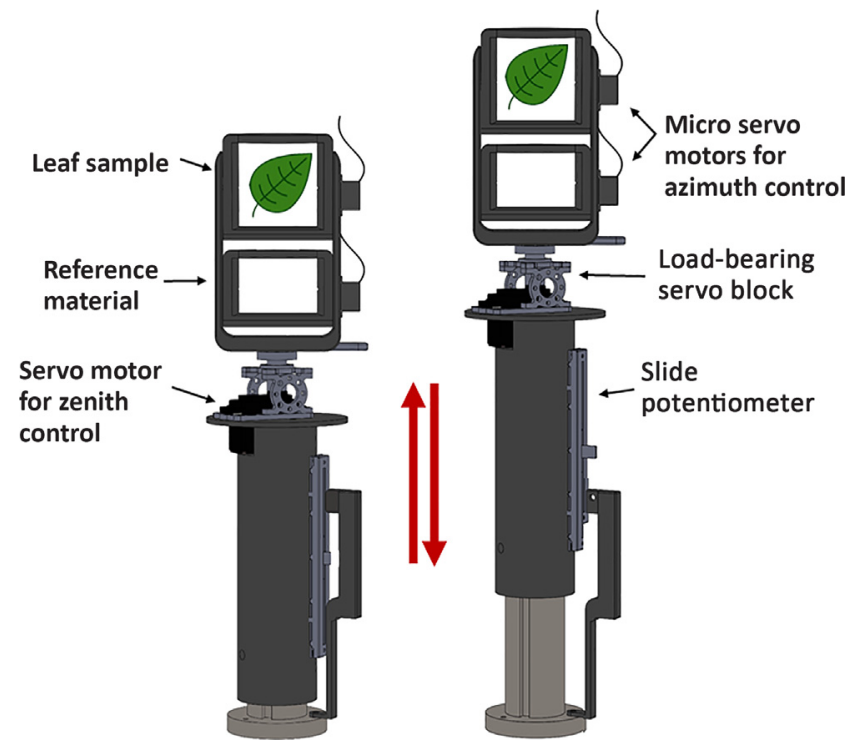

Fig. 4 CAD model of the sample holder and central pillar of the GoSPo.

A full model of GoSPo, minus its enclosure, is shown in Fig. 3. The sample pillar (Fig. 4) defines the center of the instrument. Mounted on top of the pillar, the sample holder consists of a U-shaped structure holding two rectangular frames. The top frame holds the sample, while the lower frame holds a reference target for periodic reference or calibration checks during operation. A leaf is placed in a black card-stock sleeve that is closed gently to prevent damaging the leaf and inserted into the sample holder from the top. The $\alpha_{1}$ angles of both the leaf sample and reference material are adjusted via a single servo motor mounted between the sample pillar and the U-shaped structure; two additional servo motors control the $\beta_{1}$ angles of the sample and reference frames, as shown in Fig. 4. A pneumatic system is used to raise the sample holder assembly on the pillar, placing the reference frame in the principal plane of the instrument for reference and calibration measurements.

A rotary stage with an open center hole provides the platform for the sensor head and mount for the spectrometers. This stage is driven by a stepper motor to control $\alpha_{2}$. This stage is centered on the sample pillar. The sensor can move to within $\pm 6.55 \mathrm{deg}$ of the light source without blocking the light. A sensor angle of $\alpha_{2}=0$ is considered to be aligned with the axis of the incident light beam (Fig. 5). By rotating the entire stage, there is no relative motion between the spectrometers and sensor head, which prevents any bending in the optical fibers. Any vibrations that occurred during the apparatus motion did not generate changes in the baseline greater than the associated noise in the spectra.

The entire instrument is housed in a box with a black interior to minimize stray light, and most structural components have been painted flat black for the same reason (Fig. 5).

The control software was written in Python 2.7 and coordinates the seven-degrees-of-freedom motor control and data collection from the spectrometers. A data acquisition sequence is defined by a preset list of points and integration times contained in a text file.

A series of measurement protocols were developed to correspond with the desired features to be examined. These protocols included a method for integrating measurements to estimate hemispherical reflectance and transmittance spectra, characterization of polarization at Brewster's angle, and a full characterization of the effects of different illumination angles (both with and without polarization). Other protocols were developed based on experimental design requirements and allowed for single illumination and sensor angle measurements to be taken.

A $100-\mu \mathrm{m}$-thick sheet of polytetrafluoroethylene (PTFE) was used as a reference material for calibrating both reflectance and transmittance as it provided a good distribution of light between these two measurements. This was done partially due to high levels of detector saturation that would occur if the incident light were characterized unscattered. The reference material measurements were then corrected to the known diffuse directional-hemispherical reflectance and 




(a)



(b)

Fig. 5 (a) The GoSPo in a nearly complete stage of construction and (b) a top-down view post completion. The instrument is housed in a black enclosure with a hinged lid.

transmittance spectra of the PTFE sheet as determined using a Cary 5G spectrophotometer and integrating sphere (Agilent Technologies, Mississauga, Ontario, Canada). Dark scans were collected for each sample to correct for spectrometer baseline drift and stray light. These scans were taken with the source nadir to the sample $\left(\alpha_{1}=0\right)$ and the sensor head positioned at $\alpha_{2}=90$ deg. In this position, the detector view of the sample is blocked by the side of the sample holder frame. Angle-specific measurements were corrected to the full hemispherical reflectance or transmittance reference to obtain the reflectance factor or transmittance factor.

\section{Measurements and Calibrations}

Three capabilities of GoSPo are tested from basic calibration of an application to plant leaf measurements. First, the ability of hemispherical reflectance and transmittance to be estimated is tested, with comparisons made to integrating sphere measurements. Directional reflectance and transmittance are assessed via observations of polar plots. Polarization measurements are checked against Malus' law and applied to test variations induced by leaf surface properties.

\subsection{Hemispherical Approximations}

To obtain hemispherical measurements using GoSPo, the directional reflectance and transmittance measurements were integrated over their respective azimuthal band as shown in Fig 6 with nadir illumination $\left(\theta_{I}=0\right)$. For hemispherical reflectance, the dark-corrected directional measurements between zenith angles of 0 to $\pi / 2$ and $3 \pi / 2$ to $2 \pi$ were integrated resulting in two overlapping hemispherical estimates of the same space (one from integrating 0 to $\pi / 2$ and one from integrating $3 \pi / 2$ to $2 \pi$ ). The sum of these hemispherical integrated measurements was then divided by the sum of the dark-corrected directional measurements of the reference scan integrated over the same angles [Eq. (4)].

$$
\rho=\frac{\int_{0}^{\pi / 2} 2 \pi r L\left(\theta_{E}\right)\left(\sin \left(\theta_{E}\right)\right) \mathrm{d} \theta_{E}+\int_{3 \pi / 2}^{2 \pi}-2 \pi r L\left(\theta_{E}\right)\left(\sin \left(\theta_{E}\right)\right) \mathrm{d} \theta_{E}}{\int_{0}^{\pi / 2} 2 \pi r L_{\mathrm{ref}}\left(\theta_{E}\right)\left(\sin \left(\theta_{E}\right)\right) \mathrm{d} \theta_{E}+\int_{3 \pi / 2}^{2 \pi}-2 \pi r L_{\mathrm{ref}}\left(\theta_{E}\right)\left(\sin \left(\theta_{E}\right)\right) \mathrm{d} \theta_{E}},
$$

where $\rho$ is the hemispherical reflectance, $L$ is the dark-corrected light measured at each angle, $L_{\text {ref }}$ is the dark-corrected reference light measured at each angle, and $\theta_{E}$ is the sensor angle. 
Peters, Hagey, and Noble: GoSPo: a goniospectropolarimeter to assess reflectance...

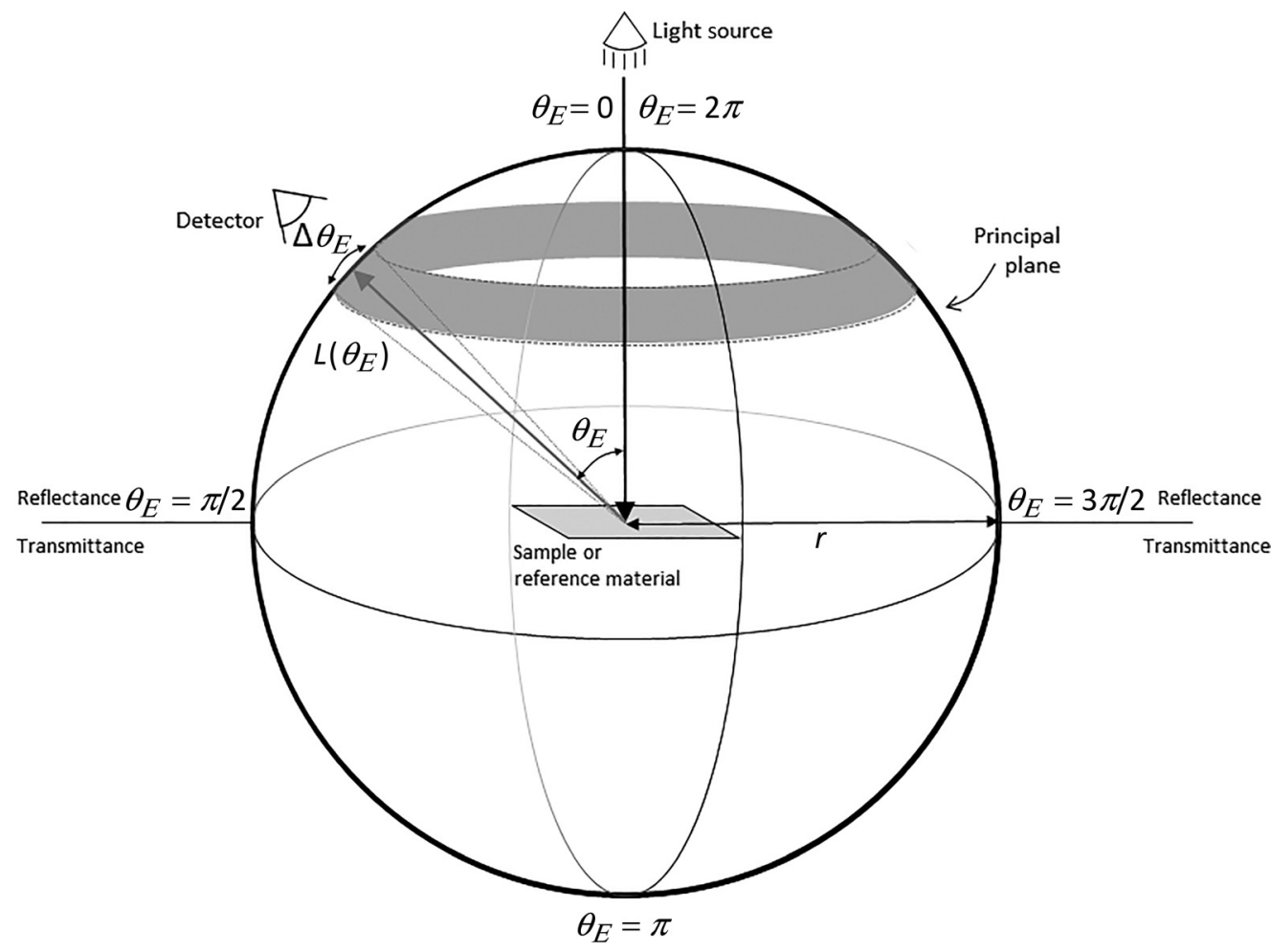

Fig. 6 Diagram showing the orientation for GoSPo's hemispherical integration.

The discretized form is shown in Eq. (5) and visualized in Fig. 6.

$$
\rho=\frac{\sum_{0}^{\pi / 2} 2 \pi r L\left(\theta_{E}\right) \sin \left(\theta_{E}\right) \Delta \theta_{E}-\sum_{3 \pi / 2}^{2 \pi} 2 \pi r L\left(\theta_{E}\right) \sin \left(\theta_{E}\right) \Delta \theta_{E}}{\sum_{0}^{\pi / 2} 2 \pi r L_{\mathrm{ref}}\left(\theta_{E}\right) \sin \left(\theta_{E}\right) \Delta \theta_{E}-\sum_{3 \pi / 2}^{2 \pi} 2 \pi r L_{\mathrm{ref}}\left(\theta_{E}\right) \sin \left(\theta_{E}\right) \Delta \theta_{E}} .
$$

This equation was simplified due to the constant radius and angle step size in sample and reference measurements as shown in Eq. (6). A similar series of equations were developed for transmittance in Eq. (7) through Eq. (9) with $\tau$ representing the hemispherical transmittance.

$$
\begin{gathered}
\rho=\frac{\sum_{0}^{\pi / 2} L\left(\theta_{E}\right) \sin \left(\theta_{E}\right)-\sum_{3 \pi / 2}^{2 \pi} L\left(\theta_{E}\right) \sin \left(\theta_{E}\right)}{\sum_{0}^{\pi / 2} L_{\mathrm{ref}}\left(\theta_{E}\right) \sin \left(\theta_{E}\right)-\sum_{3 \pi / 2}^{2 \pi} L_{\mathrm{ref}}\left(\theta_{E}\right) \sin \left(\theta_{E}\right)}, \\
\tau=\frac{\int_{\pi / 2}^{3 \pi / 2} 2 \pi r L\left(\theta_{E}\right)\left(\left|\sin \left(\theta_{E}\right)\right|\right) \mathrm{d} \theta_{E}}{\int_{\pi / 2}^{3 \pi / 2} 2 \pi r L_{\mathrm{ref}}\left(\theta_{E}\right)\left(\left|\sin \left(\theta_{E}\right)\right|\right) \mathrm{d} \theta_{E}}, \\
\tau=\frac{\sum_{\pi / 2}^{3 \pi / 2} 2 \pi r L\left(\theta_{E}\right)\left|\sin \left(\theta_{E}\right)\right| \Delta \theta_{E}}{\sum_{\pi / 2}^{3 \pi / 2} 2 \pi r L_{\mathrm{ref}}\left(\theta_{E}\right)\left|\sin \left(\theta_{E}\right)\right| \Delta \theta_{E}}, \\
\tau=\frac{\sum_{\pi / 2}^{3 \pi / 2} L\left(\theta_{E}\right)\left|\sin \left(\theta_{E}\right)\right|}{\sum_{\pi / 2}^{3 \pi / 2} L_{\mathrm{ref}}\left(\theta_{E}\right)\left|\sin \left(\theta_{E}\right)\right|} .
\end{gathered}
$$

For the hemispherical integration to be accurate, the BRDF of the sample must be uniform around the sample normal axis - an assumption that is not true for the majority of samples due to macro- and microsurface structures that alter the effective illumination angle. The potential error from this assumption is reduced by averaging the measurements from both sides of the hemisphere ( 0 to $\pi / 2$ and $3 \pi / 2$ to $2 \pi$ ). 


\subsection{Evaluation of Hemispherical Approximations}

GoSPo is capable of obtaining reflectance and transmittance measurements at subdegree intervals in the hemisphere, but this requires long scan times and may not be necessary to obtain good integrated approximations. To determine the effect of angular resolution at 1-deg and 5-deg sensor increments on reflectance and transmittance estimates, a 250- $\mu \mathrm{m}$ PTFE sheet was measured twice in GoSPo. The resulting hemispherical reflectance and transmittance spectra are compared in Fig. 7. The root-mean-square errors (RMSEs) between these spectra are 0.00649 and 0.00827 for reflectance and transmittance, respectively. For surfaces without strongly directional characteristics, a 5 deg interval was sufficient.

Figure 8 shows the resulting hemispherical approximation of three previously described PTFE sheets (thicknesses of 250,500 , and $1000 \mu \mathrm{m}$ ) when the integrations are performed with measurements taken in $5 \mathrm{deg}$ intervals from $10 \mathrm{deg}$ to $350 \mathrm{deg}$. For comparison, the diffuse reflectance and transmittance of the same PTFE sheets as measured in a Cary 5G spectrophotometer are also shown. These measurements were taken using an integrating sphere in diffuse mode and corrected with a Spectralon reflectance standard (Labsphere, North Sutton, New Hampshire). The RMSE values between these two measurements types in reflectance were $0.0827,0.0402$, and 0.1795 for 1000-, 500-, and 250- $\mu \mathrm{m}$-thick sheets, respectively. The RMSE values for transmittance were $0.1080,0.0692$, and 0.0082 for 1000-, 500-, and 250- $\mu \mathrm{m}$-thick sheets, respectively. These results indicate that given the case of a symmetrical BRDF, using discrete measurements and integrating over the hemisphere can adequately approximate hemispherical reflectance and transmittance without the use of an integrating sphere.

To investigate the accuracy of this integration in non-Lambertian samples, a common bean (Phasoleus vulgaris L.) leaf with an undulating surface (at both the micro- and macrolevels) was measured in GoSPo and compared to measurements taken in a Cary 5000 spectrophotometer (as opposed to the Cary 5G, which was used in the PTFE comparison) with an integrating sphere. The images of the leaf, the directional reflectance and transmittance, and the hemispherical comparison are shown in Fig. 9. Despite the non-Lambertian physical surface, the integrated measurements are able to match the Cary 5000 measurements with RMSE values of 0.0189 and 0.0216 for reflectance and transmittance, respectively [Fig. 9(b)], which are comparable to the reference RMSE values of the PTFE sheets (Fig. 8). The directional polar plot in Fig. 9(d) will be discussed in the next section.

\subsection{Polar Plots for Directional Reflectance and Transmittance}

To assess the ability of the instrument to obtain directional reflectance and transmittance measurements that were comparable to expected theoretical trends, PTFE sheets with thicknesses of
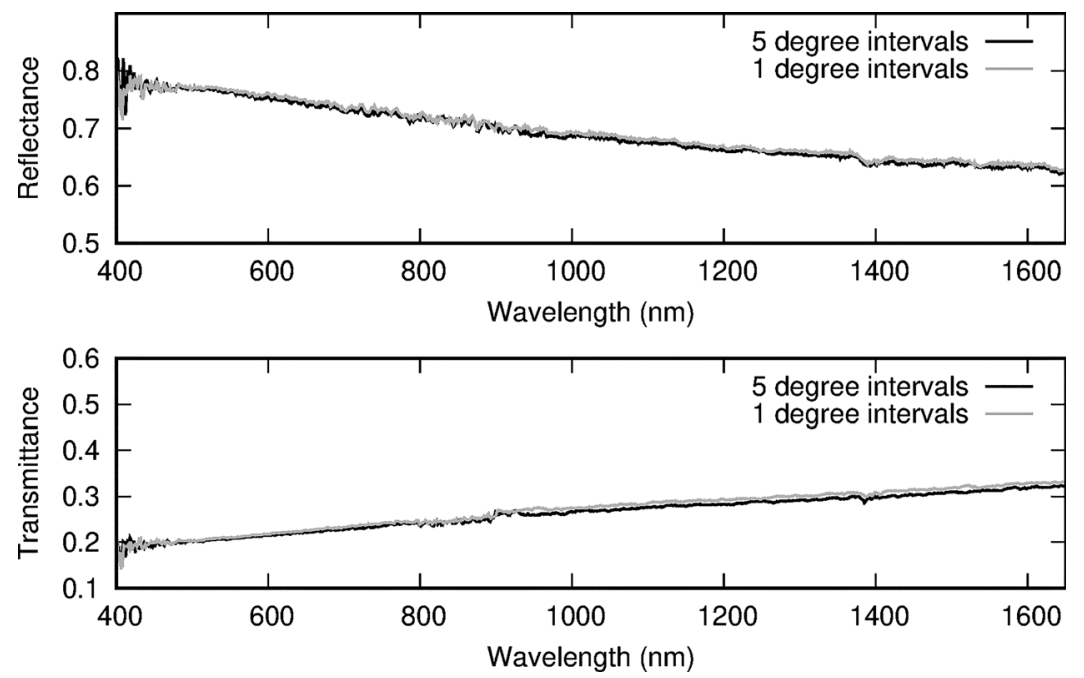

Fig. 7 Comparison of reflectance and transmittance of a PTFE sheet using 1 and 5 deg intervals for integration. 
Peters, Hagey, and Noble: GoSPo: a goniospectropolarimeter to assess reflectance...
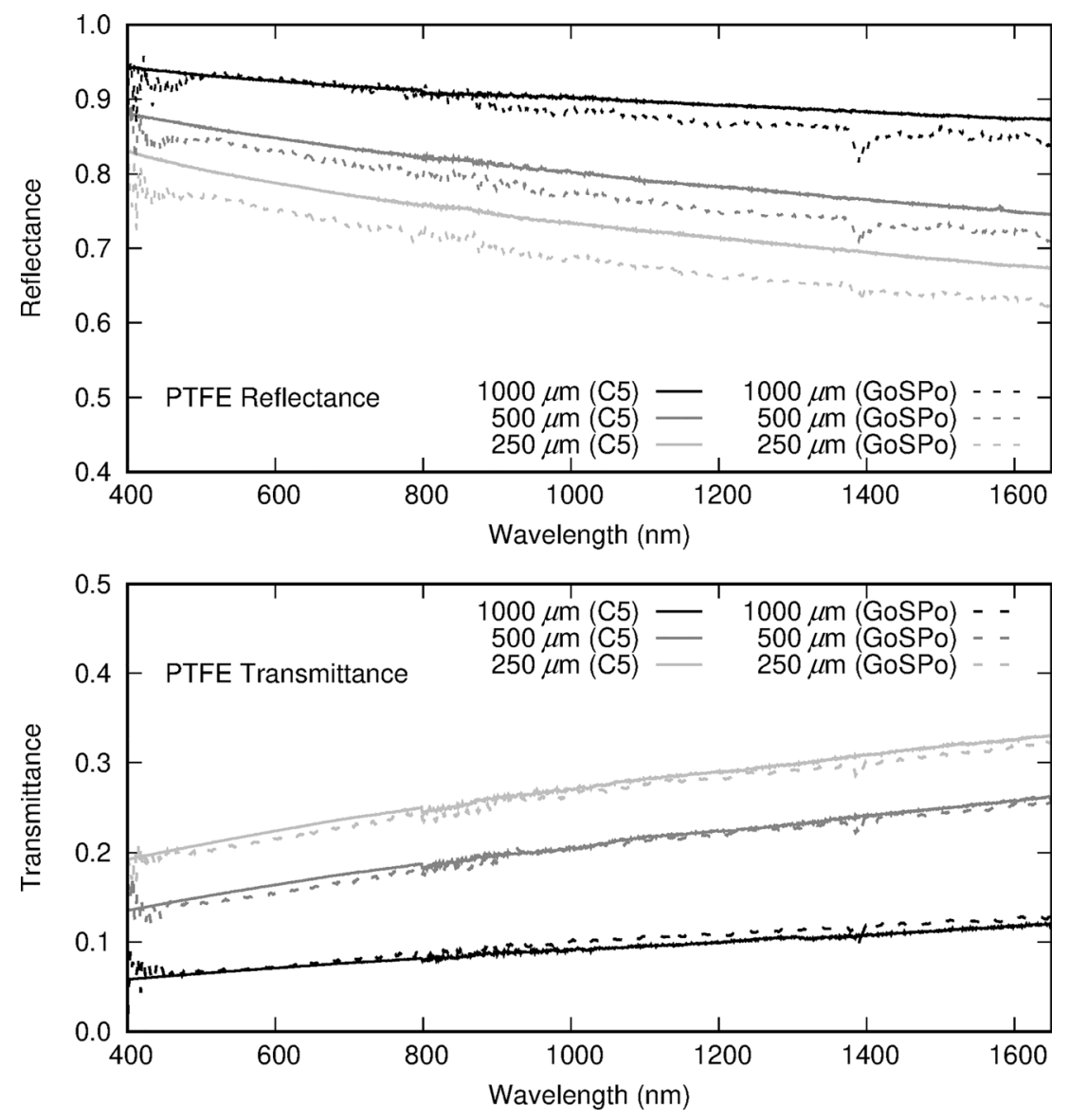

Fig. 8 Hemispherical reflectance and transmittance approximations by GoSPo as compared to the Cary $5 \mathrm{G}$.

$100,250,500$, and $1000 \mu \mathrm{m}$ were placed in the sample holder. Specular reflectance was expected to be minimal. In agreement with predictions, the scans of PTFE approached Lambertian directional characteristics for both reflectance and transmittance (Fig. 10). The PTFE sheets showed an increase in reflectance with increasing thickness and a decrease in transmittance consistent with the Beer-Lambert law. These results agree with previous studies on the optical properties of PTFE. $^{16}$

Directional spectra can assist in determining surface characteristics and can be used to link the hemispherical approximations to biconical measurements that may be possible in the field. The polar plot for the bean leaf discussed previously is shown in Fig 9(d) for three wavelengths. Multiple wavelengths are shown as the polar distribution is wavelength dependent. This information can relate to leaf biochemical and biophysical properties and can be useful in their identification. The angular distribution is not measured when using an integrating sphere.

\subsection{Polarization and Malus's Law}

To test the polarization capabilities of GoSPo, a series of transmittance measurements through both polarizing filters were collected (with no sample in the holder and the sensor directly in line with and opposite to the light source). In this measurement series, the polarizer on the light source was kept stationary while the polarizer on the sensor was rotated at 5 deg intervals over $90 \mathrm{deg}$. These results were then compared to the expected values calculated using Malus's law for two linear polarizers (Fig. 11). These experimental measurements averaged over the 500- to 900-nm band have a maximum offset of 5.9\% to the theoretical result. Using single measurements in this waveband this offset, was as high as $14.8 \%$. Although the averaged results and trend shown in Fig. 11 have good agreement with theoretical values, there is some polarization 
Peters, Hagey, and Noble: GoSPo: a goniospectropolarimeter to assess reflectance...

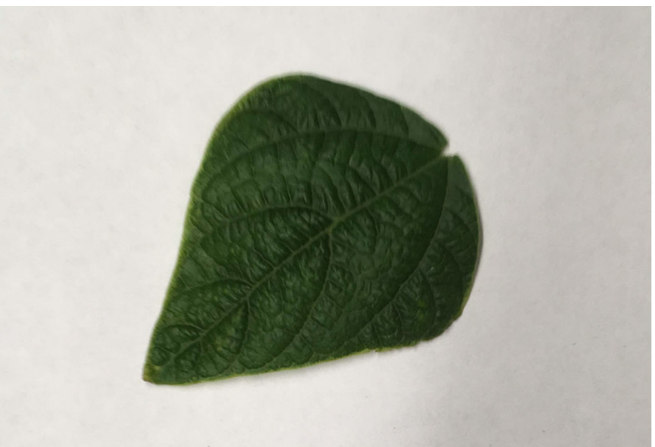

(a)

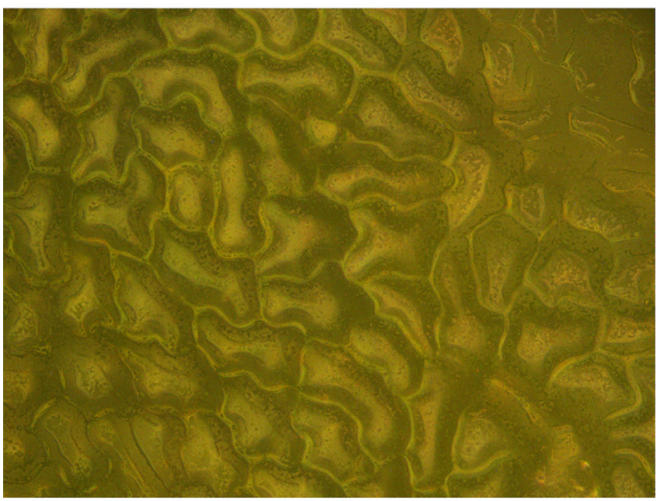

(c)



(b)

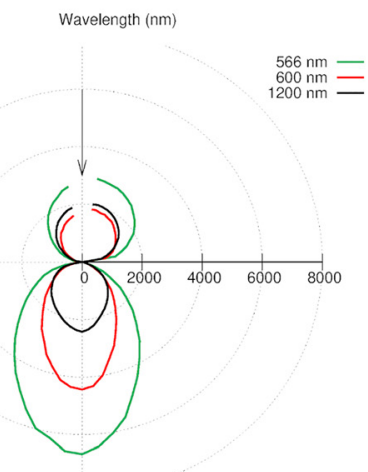

(d)

Fig. 9 Example leaf with (a) camera image; (b) hemispherical measurements from the Cary 5000 and approximation from GoSPo; (c) 500 times magnification microscopic image; (d) directional reflectance and transmittance.

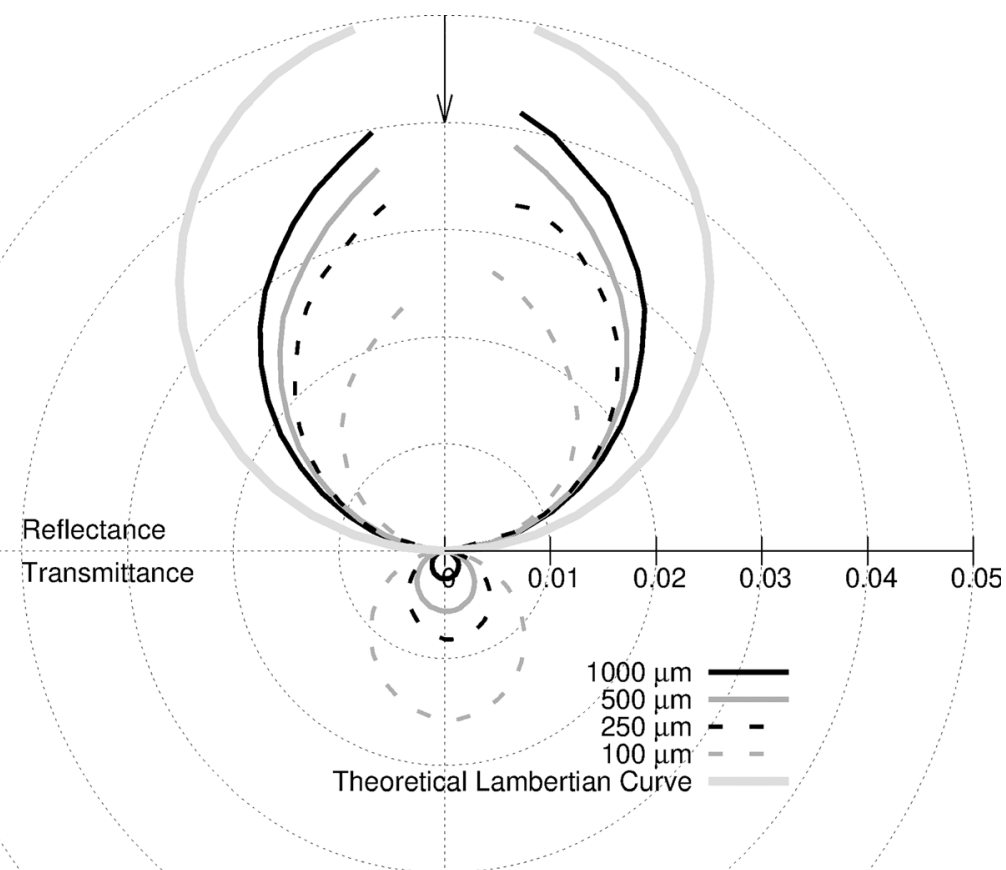

Fig. 10 Polar plots showing the reflectance and transmittance factors of PTFE sheets at $650 \mathrm{~nm}$ for four sheet thicknesses. 


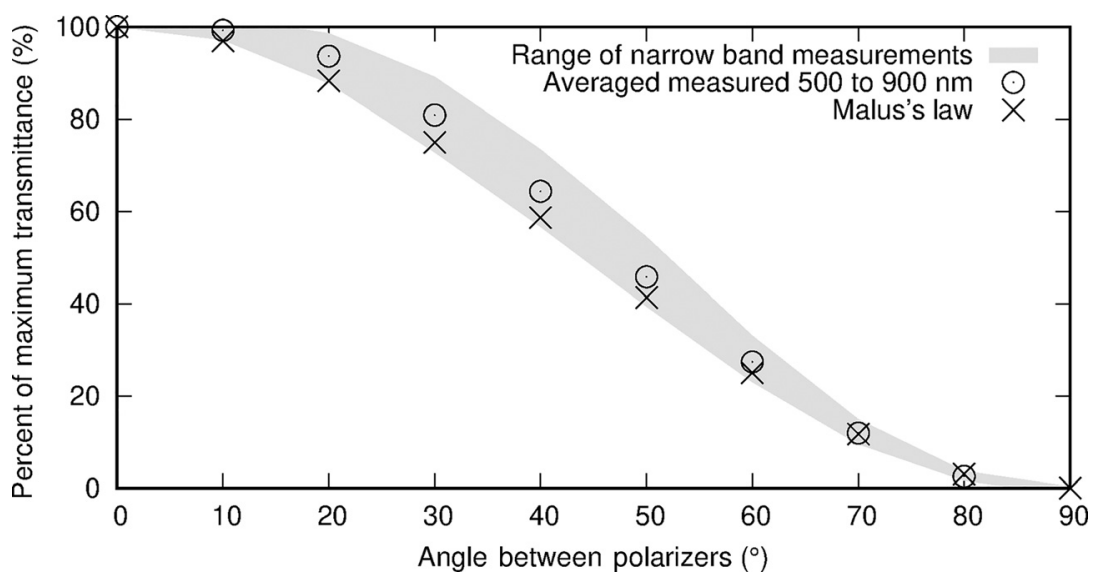

Fig. 11 Averaged transmittance measurements between 500 and $900 \mathrm{~nm}$ through two polarizing filters in GoSPo at relative angles between $0 \mathrm{deg}$ and $90 \mathrm{deg}$ compared to expected values according to Malus's law. The range of narrow band measured values between 500 and $900 \mathrm{~nm}$ is shown by the shaded area.

sensitivity associated with the spectrometers that are affecting these results. The optical fiber reduces this error by partially depolarizing the signal, but the use of a polarization scrambler would provide a more complete depolarization and reduce the error associated with the spectrometer sensitivity. The choice of a polarization scrambler is not discussed in this paper as it would be highly dependent on the application and can affect the spectral shape depending on the waveband of interest. The results discussed here indicate GoSPo's ability to selectively measure polarization orientations for broad-waveband applications, which can be useful in assessing surface properties of samples.

Four bean leaves with different surfaces were examined at Brewster's angle (approximately $\theta_{I}=55 \mathrm{deg}$ and $\theta_{E}=55 \mathrm{deg}$ for leaves ${ }^{12}$ ) while the sensor polarizer was oriented at angles of $0 \mathrm{deg}, 30 \mathrm{deg}, 60 \mathrm{deg}$, and $90 \mathrm{deg}$ relative to the plane of the sample ( $0 \mathrm{deg}$ corresponding to $S$ polarization and 90 deg corresponding to $P$ polarization). The results from these measurements are shown in Fig. 12. In this example, the rough leaf represents the leaf used in Fig. 9. The expectation with polarization variation was that a smoother, shinier surface should have a greater reflectance of specular light, and therefore, more variation with the sensor polarizer angle. This is seen in Fig. 12 as the glossy leaf has a large separation between the reflectance curves and the rough leaf has low separation. These measurements were taken concurrently with measurements for the hemispherical integration without sample disruption between scans. This allowed for hemispherical data and surface information to be obtained for the same location on a sample, which is not usually possible. In the glossy leaf example, an unexpected reflectance shape is observed, with valleys near 810 and $940 \mathrm{~nm}$; these are most notable in the $0 \mathrm{deg}$ and $30 \mathrm{deg}$ curves. These features are related to a thin-film interference resulting from a protective layer on the polarizing filters. With the previous applications discussed that use a broadband average, the impact of this effect is reduced but will need to be addressed if higher resolution spectral features are of interest. This can be accomplished using a different polarizing filter and a depolarizer. The filters used and presented in this work have a wide bandwidth (400 to $1200 \mathrm{~nm}$ ). This thin-film interference does not affect the calibration measurements presented as the polarizing filters are removed during those collections.

\section{Discussion and Conclusions}

GoSPo provides a method of approximating hemispherical measurements while obtaining angle-specific biconical light reflectance and transmittance. This is particularly valuable when applying models based on controlled laboratory measurements to field situations where hemispherical measurements are not possible. Good approximations of hemispherical reflectance and transmittance $(\mathrm{RMSE}<0.18)$ have been obtained in GoSPo when integrating measurements 
Peters, Hagey, and Noble: GoSPo: a goniospectropolarimeter to assess reflectance...
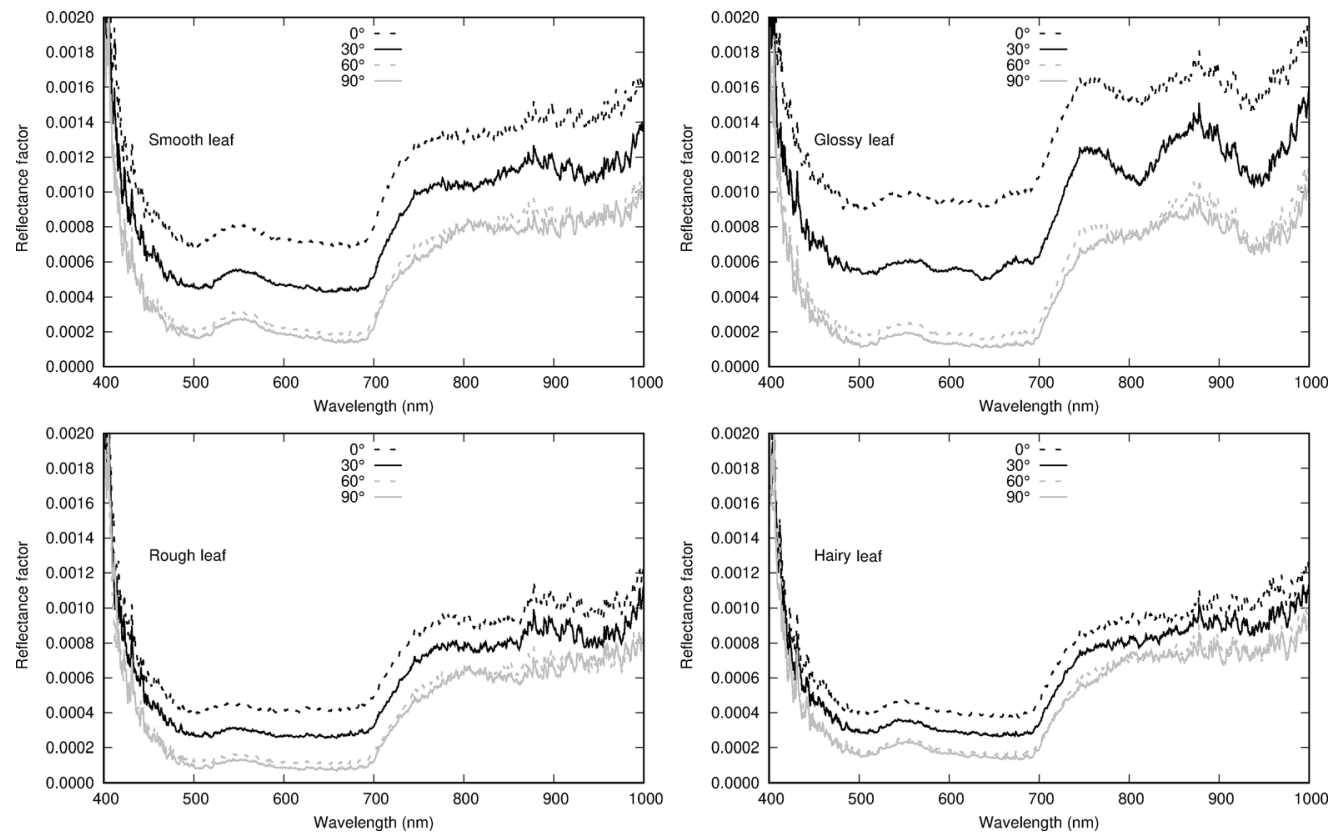

Fig. 12 Reflectance factor measured at Brewster's angle with polarization variation between sample and sensor.

collected at 5 deg intervals around the hemisphere. An advantage of GoSPo is that reflectance, transmittance, and associated reference measurements can all be acquired without sample disruption, ensuring that the same spot on the sample is assessed. The polarization filters used in this work illustrated the potential use of GoSPo for surface characterization in the leaf example, again without sample disruption. Having successfully demonstrated this potential, limitations of the current polarization elements used in this design can be improved going forward.

Future work will explore the use of polarization in GoSPo to model leaf surface characteristics. The angular distribution over the hemisphere will also be investigated to determine how non-Lambertian leaf samples are reflecting and transmitting light with the knowledge of these surface properties. Beyond agricultural applications, GoSPo could be used to assess reflectance from a variety of samples and provides a quick method for assessing VIS-NIR spectral directional distributions and polarization characteristics.

\section{Acknowledgments}

This work was supported in part by the Plant Phenotyping and Imaging Research Centre funded through the Canada First Research Excellence Fund and in part by the Natural Sciences and Engineering Reserch Council of Canada.

\section{References}

1. J.-B. Féret et al., "PROSPECT-D: towards modeling leaf optical properties through a complete lifecycle," Remote Sens. Environ. 193, 204-215 (2017).

2. D. Combes et al., "A new spectrogoniophotometer to measure leaf spectral and directional optical properties," Remote Sens. Environ. 109(1), 107-117 (2007).

3. S. Jay et al., "A physically-based model for retrieving foliar biochemistry and leaf orientation using close-range imaging spectroscopy," Remote Sens. Environ. 177, 220-236 (2016).

4. L. Bousquet et al., "Leaf BRDF measurements and model for specular and diffuse components differentiation," Remote Sens. Environ. 98(2-3), 201-211 (2005).

5. L. Bousquet et al., "Corrigendum to 'Leaf BRDF measurements and model for specular and diffuse components differentiation'," Remote Sens. Environ. 109(1), 126 (2007). 
6. V. C. Vanderbilt and L. Grant, "Plant canopy specular reflectance model," IEEE Trans. Geosci. Remote Sens. GE-23(5), 722-730 (1985).

7. L. Grant, C. S. T. Daughtry, and V. C. Vanderbilt, "Polarized and specular reflectance variation with leaf surface features," Physiol. Plant. 88(1), 1-9 (1993).

8. G. Schaepman-Strub et al., "Reflectance quantities in optical remote sensing-definitions and case studies," Remote Sens. Environ. 103(1), 27-42 (2006).

9. L. Grant, "Diffuse and specular characteristics of leaf reflectance," Remote Sens. Environ. 22(2), 309-322 (1987).

10. V. C. Vanderbilt, L. Grant, and S. L. Ustin, "Polarization of light by vegetation," in PhotonVegetation Interactions, R. B. Myneni and J. Ross, Eds., pp. 191-228, Springer, Berlin, Heidelberg (1991).

11. J. T. Woolley, "Reflectance and transmittance of light by leaves," Plant Physiol. 47(5), 656-662 (1971).

12. V. C. Vanderbilt and L. Grant, "Polarization photometer to measure bidirectional reflectance factor R $\left(55^{\circ}, 0^{\circ} ; 55^{\circ}, 180^{\circ}\right)$ of leaves," Opt. Eng. 25(4), 254566 (1986).

13. A. Comar et al., "ACT: a leaf BRDF model taking into account the azimuthal anisotropy of monocotyledonous leaf surface," Remote Sens. Environ. 143, 112-121 (2014).

14. S. R. Sandmeier and K. I. Itten, "A field goniometer system (FIGOS) for acquisition of hyperspectral BRDF data," IEEE Trans. Geosci. Remote Sens. 37(2), 978-986 (1999).

15. C. A. Coburn and S. D. Noble, "ULGS II: a high-performance field and laboratory spectrogoniometer for measuring hyperspectral bidirectional reflectance characteristics," IEEE Trans. Geosci. Remote Sens. 54(4), 2304-2313 (2016).

16. B. K. Tsai et al., "A comparison of optical properties between solid PTFE (Teflon) and (low density) sintered PTFE," Proc. SPIE 7065, 70650Y (2008).

Reisha D. Peters received her BSc and MSc degrees from the University of Saskatchewan in 2013 and 2016, respectively. She is currently pursuing her $\mathrm{PhD}$ in biological engineering at the University of Saskatchewan. Her research interests include spectroscopic analysis and computer modeling for simple, fast analysis of samples in a variety of settings and disciplines.

Simone R. Hagey received her BSc Honours in physics from the University of Saskatchewan in 2020 and will pursue an MSc degree in astronomy from the University of British Columbia with a focus in planetary science. In 2017 and 2018, she worked on the design and construction of GoSPo.

Scott D. Noble received his MSc degree from the University of Saskatchewan and his BSc and $\mathrm{PhD}$ degrees from the University of Guelph. He is an associate professor in the Department of Mechanical Engineering at the University of Saskatchewan. His current research activities include studies on pneumatic conveying in agricultural machinery, leaf optical property modeling, and applications of imaging and spectroscopy in mining, nondestructive testing, and plant phenotyping. 